\title{
Strong enhancement of transport by interaction on contact links
}

\author{
Bohr, Dan; Schmitteckert, P.
}

Published in:

Physical Review B Condensed Matter

Link to article, DOI:

10.1103/PhysRevB.75.241103

Publication date:

2007

Document Version

Publisher's PDF, also known as Version of record

Link back to DTU Orbit

Citation (APA):

Bohr, D., \& Schmitteckert, P. (2007). Strong enhancement of transport by interaction on contact links. Physical Review B Condensed Matter, 75(24), 241103. https://doi.org/10.1103/PhysRevB.75.241103

\section{General rights}

Copyright and moral rights for the publications made accessible in the public portal are retained by the authors and/or other copyright owners and it is a condition of accessing publications that users recognise and abide by the legal requirements associated with these rights.

- Users may download and print one copy of any publication from the public portal for the purpose of private study or research.

- You may not further distribute the material or use it for any profit-making activity or commercial gain

- You may freely distribute the URL identifying the publication in the public portal

If you believe that this document breaches copyright please contact us providing details, and we will remove access to the work immediately and investigate your claim 


\title{
Strong enhancement of transport by interaction on contact links
}

\author{
Dan Bohr \\ MIC, Department of Micro- and Nanotechnology, NanoDTU, Technical University of Denmark, DK-2800 Kgs. Lyngby, Denmark \\ Peter Schmitteckert \\ TKM, Institut für Theorie der Kondensierten Materie, Universität Karlsruhe, D-76128 Karlsruhe, Germany \\ (Received 7 March 2007; revised manuscript received 23 May 2007; published 12 June 2007)
}

\begin{abstract}
Strong repulsive interactions within a one-dimensional Fermi system in a two-probe configuration normally lead to a reduced off-resonance conductance. We show that if the repulsive interaction extends to the contact regions, a strong increase of the conductance may occur, even for systems where one would expect to find a reduced conductance. An essential ingredient in our calculations is a momentum-space representation of the leads, which allows a high energy resolution. Furthermore, we demonstrate that these results are independent of the high-energy cutoff and that the relevant scale is set by the Fermi velocity.
\end{abstract}

DOI: 10.1103/PhysRevB.75.241103

PACS number(s): 73.63.Kv, 73.23.Hk, 71.10.Pm

\section{INTRODUCTION}

Constructing a transport theory for strongly correlated systems is one of the major challenges of condensed matter physics. Even though many interesting ideas have been proposed during recent years, no consensus has yet emerged as to the general validity and applicability of the various schemes. With this state of affairs, it is of high importance to establish reliable benchmarks for simple model systems, which then can be used to validate new approaches.

Recently, we presented a method for calculating linear response conductance ${ }^{1}$ using the density matrix renormalization group (DMRG) method. ${ }^{2}$ A major challenge in this work consisted in minimizing finite-size effects, which was achieved via modified boundary conditions. In this paper, we circumvent these technical problems by reformulating the leads in momentum space. This approach enables us to (i) reach a much higher energy resolution $\left(\sim 10^{-5}\right)$ and (ii) allows for a greater flexibility in the choice of discretization schemes.

In two recent papers, Mehta and Andrei ${ }^{3,4}$ presented nonequilibrium Bethe ansatz results for the interacting resonantlevel model (IRLM), where a single spinless level is coupled to a left and a right lead both via a tunneling and a densitydensity interaction term. However, their work currently excludes the regime of resonant tunneling-i.e., the regime where the conductance is close to unity. ${ }^{5}$

In this work we study the linear conductance of the IRLM on a lattice to provide a benchmark for the universal properties of the model. In addition, we present results for an extended model, where the central region consists of three sites, with a similar interaction as in the IRLM model. As we will show, this model displays the same qualitative behavior as the IRLM. It should be noted that despite its simplicity, the IRLM captures much of the physics of transport through an arbitrary interacting nanostructure provided that only a single level is close to the Fermi energy of the leads, with all other levels well separated and outside the voltage window within which the transport takes place. For perfect coupling, the IRLM model corresponds to the one-dimensional model studied by Vasseur et al. ${ }^{6}$ and Molina et al., ${ }^{7}$ obtained by restricting their nanostructure to a single site. Using the embedding method, they showed that smoothing the ramp of interaction for perfect contacts can compensate for the decrease of transmission due to interaction on the nanostructure. Here we go far beyond the energy resolution attained in previous work and show that interaction on the contact links can lead to strong renormalization effects, enhancing transport beyond the noninteracting system.

\section{METHOD AND MODELS}

We use the DMRG method to evaluate the linear response conductance of the interacting nanostructure. In previous work, ${ }^{1}$ the leads were modeled in real space by nearestneighbor hopping chains. While simple to implement, there are several drawbacks of this method, most prominently the need for "damped boundary conditions" and the resulting problem of trapping of fermions on the Wilson chain (the damped region). ${ }^{1}$

In the present work, we introduce a setup where the leads are described in momentum space. Specifically, a short part of the lead close to the nanostructure is represented in real space, accounting for local (i.e., high-energy) physics, while farther away from the nanostructure the lead is represented in momentum space; see Fig. 1. Since the low-energy modes of the momentum leads are now directly coupled to the extended structure (the nanostructure plus additional real-space

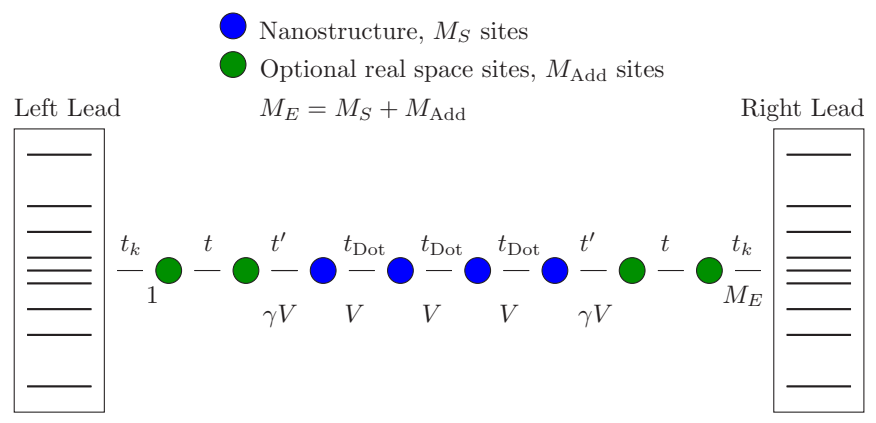

FIG. 1. (Color online) Schematics of the nanostructure extended by real-space sites and attached to momentum-space leads. 


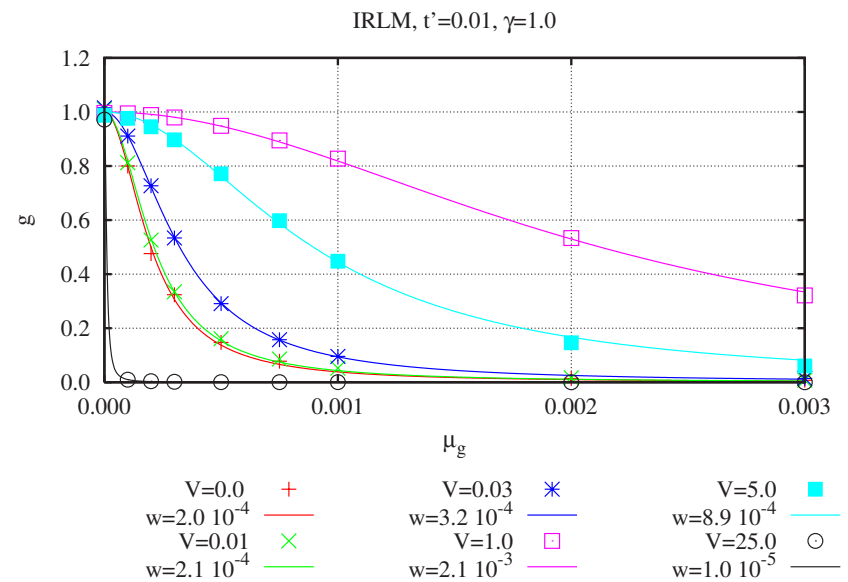

(a)

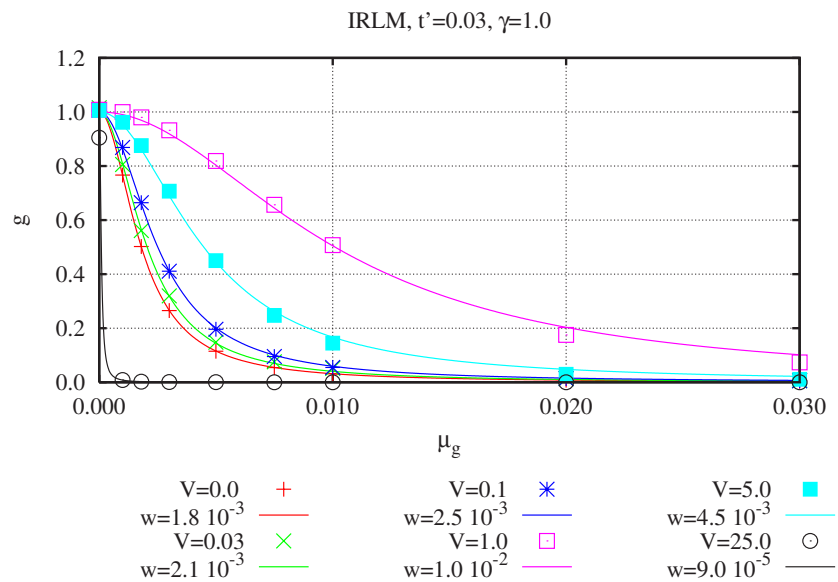

(b)

FIG. 2. (Color online) Conductance versus gate potential for the interacting resonant-level model for a contact hopping of (a) $t^{\prime}=0.01$ and (b) $t^{\prime}=0.03$ and contact interaction ranging from zero to 25 . To each set of DMRG data, a Lorentzian of half width $2 w$ has been added as a guide to the eye. The leads are described with a cosine band between \pm 2 such that the Fermi velocity is $v_{F}=2$. In contrast to intradot interaction, the contact interaction enhances the conductance and shows a nonmonotonic behavior versus contact interaction.

sites), as illustrated in Fig. 1, the trapping of fermions on the low-energy sites $^{1}$ is avoided and no scaling sweeps are needed. This enables much higher energy resolution, and in the current work we resolve resonances of widths $\mathcal{O}\left(10^{-5}\right)$.

By virtue of the momentum representation of the leads, the discretization scheme can be chosen arbitrarily to suit the problem at hand. In the present work, we use a logarithmic discretization to cover a large energy range, while switching to a linear discretization for the lowest-energy states in order to describe Fermi-surface physics accurately. The linear discretization on the low-energy scale allows for a better representation of the low-energy physics relevant for transport properties-i.e., excitations created by $\eta$.

The models considered in this work are the IRLM and the natural extension of this model to resonant linear chains, defined by the Hamiltonians

$$
\begin{gathered}
H_{R S}=\sum_{j \in S} \mu_{g} \hat{c}_{j}^{\dagger} \hat{c}_{j}-\sum_{j, j-1 \in S_{E}}\left(t_{j} \hat{c}_{j}^{\dagger} \hat{c}_{j-1}+\text { H.c. }\right) \\
+\sum_{j, j-1 \in S_{E}} V_{j}\left(\hat{n}_{j}-\frac{1}{2}\right)\left(\hat{n}_{j-1}-\frac{1}{2}\right), \\
H_{M S}=\sum_{i \in L, R} \epsilon_{i} \hat{c}_{i}^{\dagger} \hat{c}_{i}, \\
H_{T}=-\left(\sum_{k \in L} t_{k} \hat{c}_{k}^{\dagger} \hat{c}_{1}+\sum_{k \in R} t_{k} \hat{c}_{k}^{\dagger} \hat{c}_{M_{E}}\right)+\text { H.c. }
\end{gathered}
$$

where $\hat{c}_{\ell}^{\dagger}$ and $\hat{c}_{\ell}$ are the (spinless) fermionic creation and annihilation operators at site $\ell, \hat{n}_{\ell}=\hat{c}_{\ell}^{\dagger} \hat{c}_{\ell} \cdot H_{R S}, H_{M S}$, and $H_{T}$ denote real space, momentum space, and tunneling between real- and momentum-space Hamiltonians, respectively. The symbols $S$ and $S_{E}$ denote the nanostructure and the extended nanostructure (the full real-space chain), respectively. The indices 1 and $M_{E}$ denote the first and last sites in $S_{E}$. The general setup and the specific values of the hopping matrix elements $t_{j}$ and the interactions $V_{j}$ are indicated in Fig. 1, and note specifically the interactions on the contact links, $\gamma V$. The coupling $t_{k}$ of the extended real-space structure to the momentum leads is chosen in such a way that in the case of a cosine band it corresponds to a nearest-neighbor hopping chain in real space with a hopping parameter of $t$. In the following we measure all energies in units of $t=1$.

For a single-site nanostructure and $\gamma=1$ this model reduces to the IRLM. The properties of the leads are defined by the band structure $\epsilon_{k}$, which can take any form. In this work we use either the cosine band, $\epsilon_{k}=-2 \cos (k)$, or the linear band, $\epsilon_{k}=2 k$. $D$ is a cutoff parameter such that the Fermi velocity $v_{F}=2$ is kept constant in all work presented here, and the band ranges between energies $-D$ and $D$. Throughout this work we use the notion of "contact interaction" for interaction on the link between the nanostructure and the leads.

\section{RESULTS}

The aim of this work is to study the effect of contact interaction. It is known from previous work ${ }^{1}$ that strong repulsive interactions within the nanostructure lead to suppression of the transport off resonance due to the formation of a density-wave-like state on the dot.

In Fig. 2, we show results for the conductance versus gate potential for different couplings to the leads and different contact interactions for the IRLM $(\gamma=1)$. The calculations have been performed with typically 130 sites in total, $M_{E}$ $=10$ real-space sites and 120 momentum-space sites. Due to the symmetry of the band, we use a discretization that is symmetric around $\epsilon_{F}=0$, and further use identical discretization of the two leads. To represent the "large" energy span in the band we use 20 logarithmically scaled sites, and thereafter use 10 linearly spaced sites to represent the low-energy 


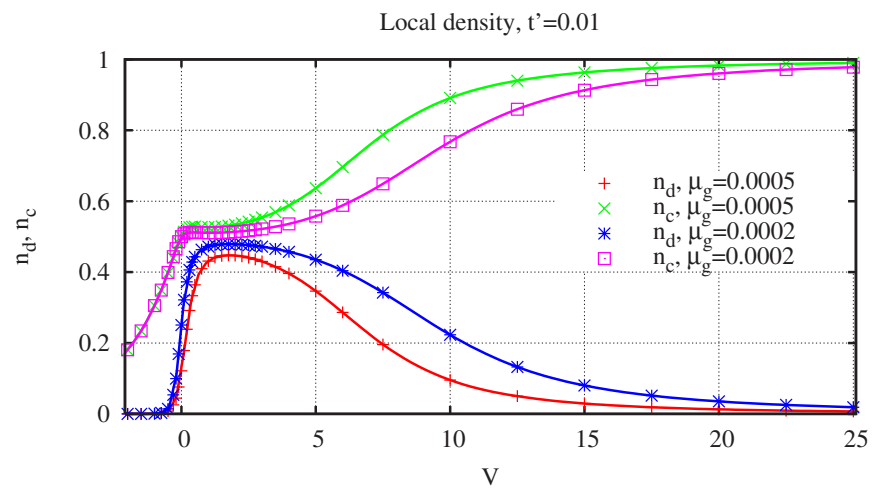

FIG. 3. (Color online) Site occupation $n_{d}$ of the resonant level and $n_{c}$ of the real-space sites attached to the level vs the link interaction in the IRLM for $t^{\prime}=0.01$ and two different gate voltages.

scale correctly. In the DMRG calculations presented we used at least 1300 states per block and 10 finite lattice sweeps. To each set of DMRG results in Fig. 2 is added a Lorentzian of half width $2 w$ as a guide to the eye.

As the interaction is turned up the width of the resonance is increased far beyond the noninteracting result, up to an order of magnitude larger; e.g., for $t^{\prime}=0.01$ and $V=1$ the resonance width is increased by a factor of 10 . However, for a larger interaction $V>v_{F}=2$, transport is suppressed, and for very large interactions the width even becomes smaller than the noninteracting resonance. A similar nonmonotonic behavior is observed by Borda et al. ${ }^{8}$ using a perturbative calculation and is opposite to the one originally reported by Mehta and Andrei, ${ }^{3}$ which, however, has been corrected in an erratum. ${ }^{5}$ Where preceding work ${ }^{3-5,8}$ failed to reach the unitary limit, we demonstrate that indeed the resonant value remains unitary.

Furthermore, by changing the bandwidth $D$ for linear bands we have verified that the relevant energy scale is the Fermi velocity $v_{F}$ of the leads, while the bandwidth $D$ does not influence the conductance, as long as $D \gg V$; compare Fig. 5.

Borda et al. ${ }^{8}$ conclude in their work that "in the case of repulsive interaction the site next to the occupied $d$ level is empty and thus that electron can easily jump to the conduction band," while for attractive interaction fermions accumulate close to the impurity. From that reasoning we would expect an asymmetric conductance curve depending on whether the impurity is filled or depleted. However, this would violate particle-hole symmetry of the model. In Fig. 3, we plot the site occupation $n_{d}$ of the resonant level and the averaged site occupation $n_{c}$ of the left and right real-space sites attached to the level. The occupations are plotted versus the contact link interaction for the interacting resonant-level model and for two different gate voltages. The site occupation of the resonant level and the neighboring sites are both enhanced by the repulsive interaction as long as interaction is in the range that enhances the conductance. For stronger interaction the site occupancy of the resonant level is indeed reduced; however, this is the regime where the conductance is reduced. We would like to remark that in the noninteracting case and for a weak contact, $t^{\prime} \ll 1$, the site occupations

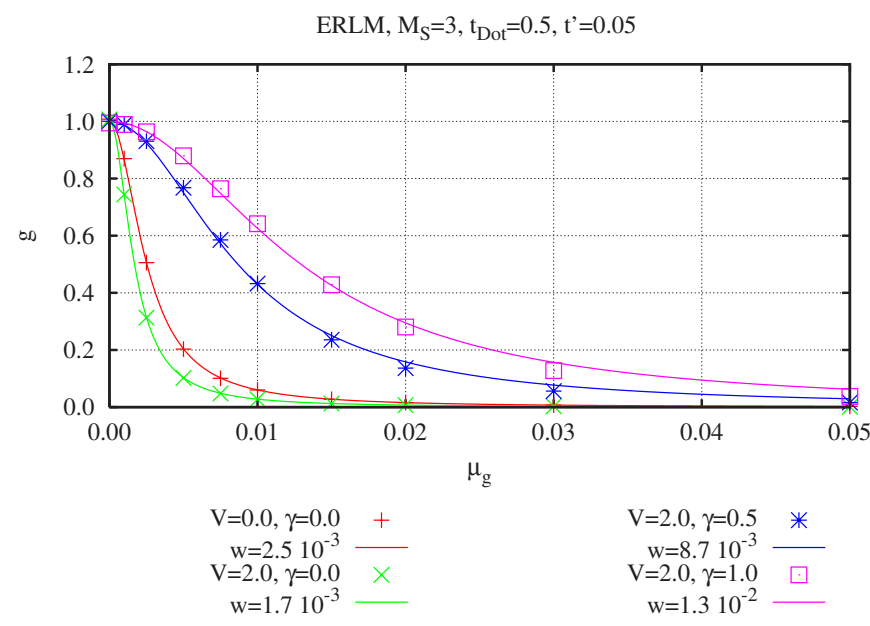

FIG. 4. (Color online) Conductance versus gate potential for a resonant three site chain. To each set of DMRG results a Lorentzian of half width $2 w$ has been added as a guide to the eye. The leads are described by a cosine band between \pm 2 such that $v_{F}=2$. The interdot interaction suppresses the transport while the contact interaction is seen to enhance the transport.

of the real-space sites in the leads change only slightly with gate voltage and are all very close to half filling. Thus it seems that the densities of the hybridizing lead levels are not the determining quantity for the interaction-induced changes of transport properties.

The strong renormalization of the resonance width and the nonmonotonic behavior is, however, not specific to the IRLM. In Fig. 4, we show results for the center peak of a three-site nanostructure. Without a contact interaction we find that the intradot interaction $V=2.0=4 t_{\mathrm{dot}}$ leads to a suppression of the transport in agreement with previous results. ${ }^{1}$ As in the single-level case already a small contact interaction increases again the width of the resonance at zero gate potential. The enhancement of the conductance by a contact interaction is stronger than the corresponding suppression by the intradot interaction. Therefore, we conjecture that the enhancement of conductance due to the contact interaction is a universal feature, which should also be present in other systems. These findings may also be relevant for disordered structures, where repulsive interaction was found to enhance transport in the case of strong disorder. ${ }^{9}$

Finally, we have considered a non-particle-holesymmetric IRLM model to address the question of parameter renormalization versus bandwidth cutoff. The non-particlehole-symmetric model is defined by replacing the $\left(\hat{n}_{j}-\frac{1}{2}\right)$ terms in $H_{R S}$ by $\hat{n}_{j}$. The results are shown in Fig. 5. It is clearly seen from the calculation that varying the cutoff over an order of magnitude does not change the resonance, providing the interaction is not cut off by the band. Neither the position nor the width of the resonance peak is influenced by the change of the cutoff $D$, which is in contrast to the the renormalization group flow that follows from the nonequilibrium Bethe ansatz. ${ }^{3}$ There, all transport quantities depend on the cutoff $D$ and the conductance changes with the cutoff. While it is often difficult to compare a field theoretical model, like the IRLM of Mehta and Andrei, with a lattice 


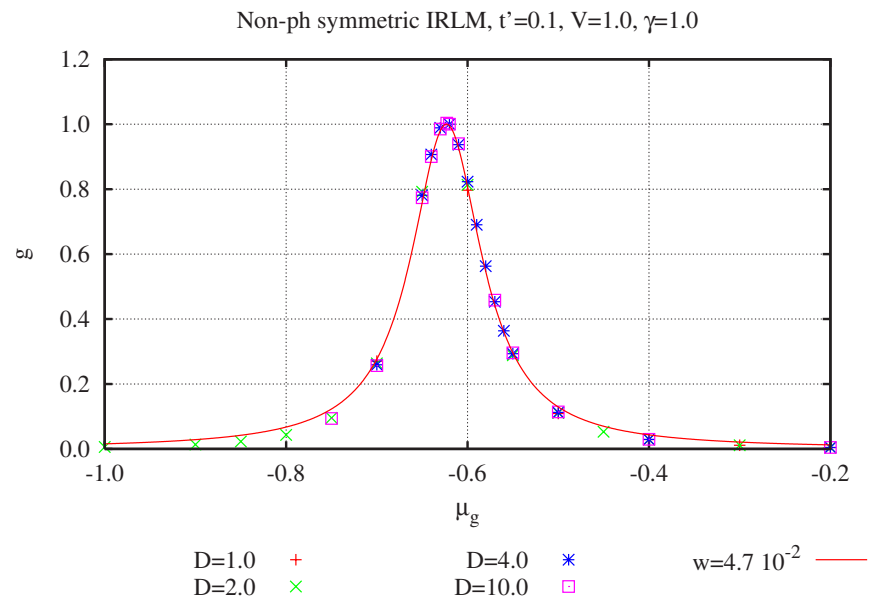

FIG. 5. (Color online) Conductance versus gate potential for a single-site nanostructure without particle-hole symmetry with a contact interaction of $V=1.0$ and a contact hopping of $t^{\prime}=0.1$ for a linear band with cutoff parameter $D=1.0,2.0,4.0$, and 10.0 and constant Fermi velocity, $v_{F}=2$. The conductance is independent of the cutoff. The solid line is a fit with a Lorentzian of half width $w=4.7 \times 10^{-2}$.

model, we can at least conclude that the RG flow found in their work is absent in our model with regularized (tight binding) leads and that the relevant energy scale is the Fermi velocity.

\section{SUMMARY}

A normal paradigm in transport calculations is to make a principal division between transport region, the nanostructure or "molecule," and leads, where all correlation effects are excluded from the leads.

In this work, we have investigated the influence of an interaction on the contact between a nanostructure and the leads in a simple tight-binding model. Using the nonperturbative DMRG method to evaluate the linear conductance we have demonstrated that a contact interaction significantly influences the transport properties. A repulsive interaction smaller or comparable to the Fermi velocity in the leads enhances the conductance, while a large interaction leads to a suppression of the conductance. Our work shows that even a slight spread of the interaction on the contacts influences the transport strongly. This demonstrates that particular care should be taken in treating the contacts correctly, especially regarding the interaction.

\section{ACKNOWLEDGMENTS}

D.B. acknowledges support from the HPC-EUROPA under Project No. RII3-CT-2003-506079, supported by the European Commission. This work also profited from Project 710 of the Landesstiftung Baden-Württemberg and partial support through project B2.10 of the DFG Center for Functional Nanostructures. Parts of the computations were performed on the XC1 and XC2 at the SSC Karlsruhe.
${ }^{1}$ D. Bohr, P. Schmitteckert, and P. Wölfle, Europhys. Lett. 73, 246 (2006).

${ }^{2}$ S. R. White, Phys. Rev. Lett. 69, 2863 (1992).

${ }^{3}$ P. Mehta and N. Andrei, Phys. Rev. Lett. 96, 216802 (2006).

${ }^{4}$ P. Mehta and N. Andrei, arXiv:cond-mat/0702612 (unpublished).

${ }^{5}$ P. Mehta, S. P. Chao, and N. Andrei, arXiv:cond-mat/0703426 (unpublished).

${ }^{6}$ G. Vasseur, D. Weinmann, and R. A. Jalabert, Eur. Phys. J. B 51,
267 (2006).

${ }^{7}$ R. A. Molina, D. Weinmann, R. A. Jalabert, G.-L. Ingold, and J.-L. Pichard, Phys. Rev. B 67, 235306 (2003).

${ }^{8}$ L. Borda, K. Vladár, and A. Zawadowski, Phys. Rev. B 75, 125107 (2007).

${ }^{9}$ R. A. Molina, P. Schmitteckert, D. Weinmann, R. A. Jalabert, G.-L. Ingold, and J.-L. Pichard, Eur. Phys. J. B 39, 107 (2004). 